\title{
UNCERTAINTY IN SPATIALLY EXPLICIT ANIMAL DISPERSAL MODELS
}

\author{
Wolf M. Mooij ${ }^{1,3}$ And Donald L. DeAngelis ${ }^{2}$ \\ ${ }^{1}$ Netherlands Institute of Ecology, Centre for Limnology, Rijksstraatweg 6, 3631 AC Nieuwersluis, The Netherlands \\ ${ }^{2}$ U.S. Geological Survey/Biological Resources Division, Department of Biology, University of Miami, \\ Coral Gables, Florida 33124 USA
}

\begin{abstract}
Uncertainty in estimates of survival of dispersing animals is a vexing difficulty in conservation biology. The current notion is that this uncertainty decreases the usefulness of spatially explicit population models in particular. We examined this problem by comparing dispersal models of three levels of complexity: (1) an event-based binomial model that considers only the occurrence of mortality or arrival, (2) a temporally explicit exponential model that employs mortality and arrival rates, and (3) a spatially explicit gridwalk model that simulates the movement of animals through an artificial landscape. Each model was fitted to the same set of field data. A first objective of the paper is to illustrate how the maximum-likelihood method can be used in all three cases to estimate the means and confidence limits for the relevant model parameters, given a particular set of data on dispersal survival. Using this framework we show that the structure of the uncertainty for all three models is strikingly similar. In fact, the results of our unified approach imply that spatially explicit dispersal models, which take advantage of information on landscape details, suffer less from uncertainly than do simpler models. Moreover, we show that the proposed strategy of model development safeguards one from error propagation in these more complex models. Finally, our approach shows that all models related to animal dispersal, ranging from simple to complex, can be related in a hierarchical fashion, so that the various approaches to modeling such dispersal can be viewed from a unified perspective.

Key words: dispersal models, uncertainty; dispersal mortality; dispersal success; individualbased modeling; landscape details; likelihood, maximum; managing endangered species; model complexity; population models; random grid-walk models; spatially explicit modeling; suitable habitat.
\end{abstract}

\section{INTRODUCTION}

Spatially explicit population models are important tools in conservation studies (Pulliam et al. 1992, Vos et al. 2001). In such studies one wants to predict the dynamics of endangered population(s) under a given set of management scenarios (Turner et al. 1995). A model that captures the essentials of the dynamics of the population(s) under study seems a prerequisite for making trustworthy predictions about future developments. One such essential component is the spatial heterogeneity of natural landscapes (Turner et al. 1993). Spatially explicit, grid-based models can take advantage of the details provided by GIS databases. Recent studies have shown that such models can be parameterized and tested in a realistic conservation biological context (Hanski 1999, McCarthy et al. 2000, Lindenmayer et al. 1999).

At the same time, however, spatially explicit population models have been heavily criticized for needing an excessive amount of biological data. Estimation of parameters regarding dispersal survival and success is difficult, and, as dispersal success is an important parameter in many populations (e.g., spotted owls;

Manuscript received 1 April 2002; revised 16 September 2002; accepted 28 September 2002; final version received 8 November 2002. Corresponding Editor: D. Lindenmayer.

${ }^{3}$ E-mail: w.mooij@nioo.knaw.nl
McKelvey et al. 1993, Franklin et al. 2000), spatially explicit models of these populations may be prone to error propagation (Ruckelshaus et al. 1997). In the face of proliferating individual-based spatially explicit models, Bart (1995) stressed that the reliability of such models needed to be better understood to be suitable for application to management issues. Others have argued that simpler, more general models or indicators of population viability on a landscape may give more reliable results (Doak and Mills 1994); and examples of these, such as the ecologically scaled landscape indices (Vos et al. 2001), may be useful in making management decisions.

Recent progress in model development and theory shows promise in allowing better parameter estimation in spatially explicit population models. Mark-recapture methods have been extended to determining dispersal parameters (e.g., Hanski et al. 2000). Also, approaches for extracting information from population patterns in space, which can help refine parameter estimates, have been developed (e.g., Grimm et al. 1996, T. Wiegand, F. Knauer, P. Kaczensky, and J. Naves, unpublished manscript). Further, Mooij and DeAngelis (1999) and South (1999) showed that error propagation in spatially explicit population models need not be as great as was previously reported in Ruckelshaus et al. (1997). Nonetheless, the suggestion that these models need an ex- 
TABLE 1. Mathematical equations for the binomial model.

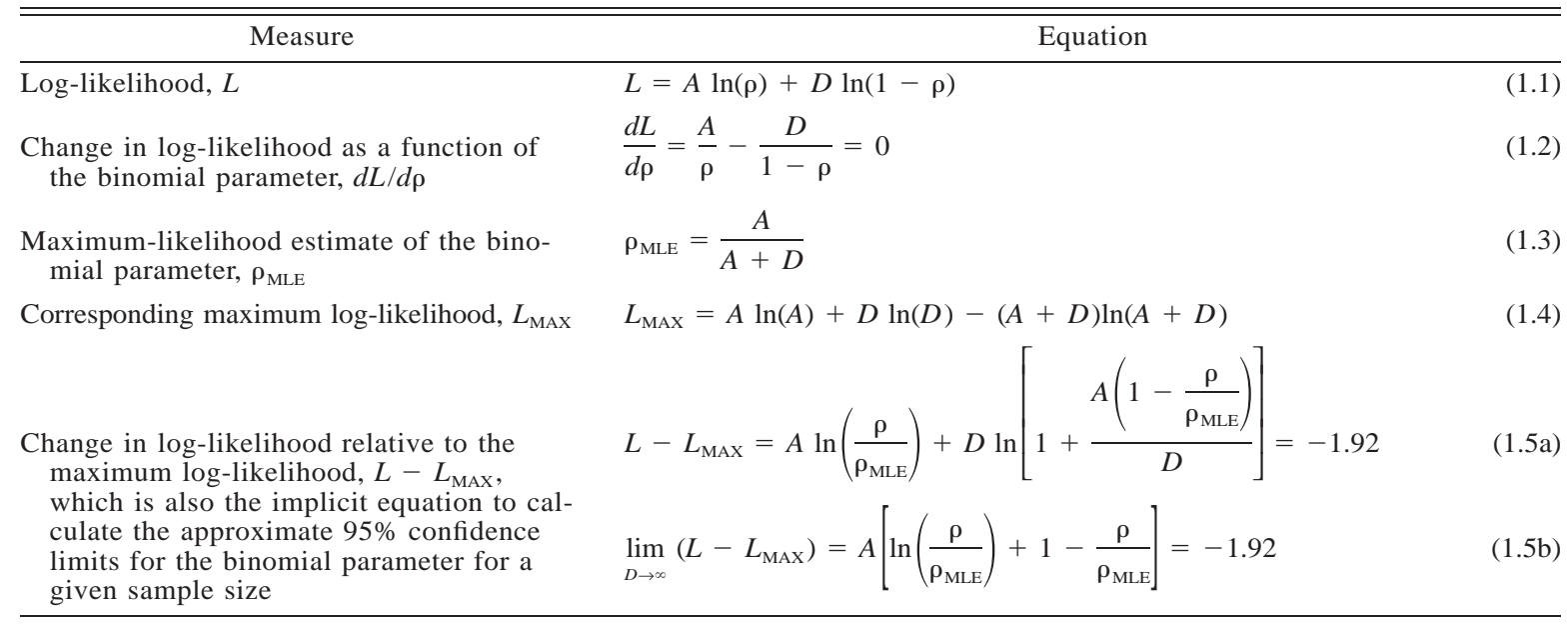

cessive amount of field data, especially when it comes to estimating dispersal success, remains unchallenged.

The aim of this paper is to address the issue of uncertainty in spatially explicit animal-dispersal models due to data limitations such as those mentioned by Ruckelshaus et al. (1999). We agree with Ruckelshaus et al. that, in light of habitat fragmentation, dispersal is a highly relevant process for which good data may be hard to obtain. We know of no pertinent analysis, however, examining whether a spatially explicit model for dispersing animals, incorporating relevant landscape detail, is necessarily less reliable than a simple dispersal model, based on the same data on the animals, but omitting spatial detail. We carry out such an analysis, using a published set of data on the survival of dispersing animals. In particular we used a field study on 24 radio-tagged Mexican Spotted Owls (Ganey et al. 1998). Ruckelshaus et al. (1999) use this paper as an example of what amount of data is typically available. We discuss our findings to draw conclusions about parameter uncertainty in spatially explicit models in general.

We applied three models of animal dispersal to Ganey et al.'s (1998) Mexican Spotted Owl data that vary widely in their level of complexity, to see if they differ in the efficiency by which they can extract information from the available data. The first, and simplest model merely uses the data on final numbers of animals in the categories of known arrivals and deaths out of the original numbers of radio-tagged animals. A binomial model is used in this case. The second model also exploits the temporal structure of the data, in which case the two parameters of interest are the probabilities of mortality and of successful arrival in a patch. An exponential model is used in this case. The third level of complexity supposes that information on the spatial configuration of habitat patches is available. Then the two parameters that need to be estimated from the field data are the probability of mortality and the rate at which the landscape is explored by the organism. For this last case a random grid-walk model is used. Thus the models increase in complexity from an "eventbased" description to a "temporally explicit" description, and finally to a "spatially and temporally explicit" description of the dispersal process. This approach of starting with a model with the minimum of resolution, and proceeding to more complex articulations of the system, is similar to the "scaling-down" strategy in modeling advocated by Thulke et al. (1999).

For each model type, we asked three basic questions: (1) How do we estimate the parameters of interest? (2) What is the degree of confidence in these parameters? (3) If the confidence limits are deemed to be too wide, can we specify what the minimum size of a data set should be, given a desired level of confidence? For all three models we answered these questions with a maximum-likelihood analysis. Using this common framework, we were able to show that there are no fundamental differences in the structure of uncertainty in these models.

\section{Methods}

Below we describe the methods that we applied to analyze, in turn, the event-based model (for equations see Table 1), the temporally explicit model (Table 2), and the spatially and temporally explicit model (Table 3 ). Each of these models can be constructed from the same set of empirical data. In order to make our description of these models as concrete as possible, we will refer to a particular data set, that of Ganey et al. (1998) on Mexican Spotted Owls. But our methods are entirely general and should apply to any similar set of data.

Ganey et al. (1998) radio-tagged 24 juvenile Mexican Spotted Owls in their natal sites in northern Arizona (USA). Of these 24 organisms, 19 initiated dis- 
TABLE 2. Mathematical equations for the exponential model.

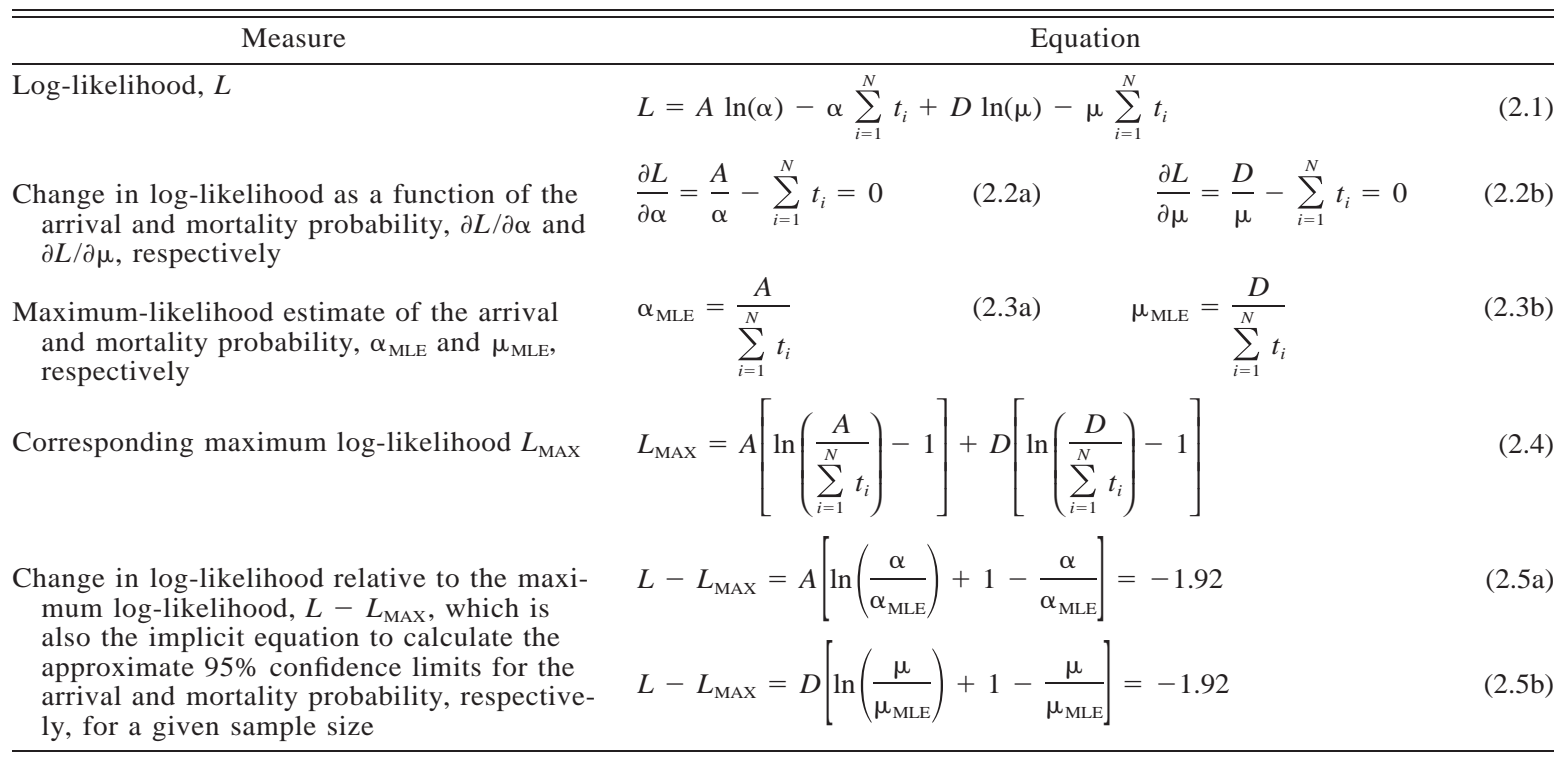

persal. By the end of the study, there was 1 survivor, 8 birds were confirmed dead, and 10 had unknown fates. The one survivor was observed breeding later on and was thus confirmed to be a successful disperser. The field data for these 19 birds, taken directly from Ganey et al. are given in Table 4. The study of Ganey is representative of the amount and type of data that will usually confront a modeler of dispersing animals (Ruckelshaus et al. 1999) and therefore serves as a good example in our analysis of the inherent uncertainty of spatially explicit population models.
Using these data we first calculated the parameters of the three models using the maximum-likelihood approach and then determined confidence limits for these parameters. Finally, we assessed what the confidence limits would be if a larger sample were available, while preserving the proportions of birds in each of Ganey et al.'s (1998) categories. To estimate best-fitting parameters and their approximate confidence limits one needs five equations for each model (Tables 1, 2, and $3)$. Each of these equations has a general interpretation and this approach should apply to any data set and any

TABLE 3. Mathematical equations for the random grid-walk model.

\begin{tabular}{|c|c|c|c|}
\hline Measure & Equation & & \\
\hline Log-likelihood, $L$ & $\begin{aligned} L & =\sum_{i=1}^{A} \ln \left(\beta a_{\beta t_{i}}\right)+\sum_{i=1}^{N} \ln \left(s_{\beta t_{i}}\right) \\
L & =a+b \ln (\beta)+c \ln ^{2}(\beta)+d \ln ^{3}(\beta)\end{aligned}$ & & $\begin{array}{l}(3.1 \mathrm{a}) \\
(3.1 \mathrm{~b})\end{array}$ \\
\hline $\begin{array}{l}\text { Change in log-likelihood as a function of the } \\
\text { logarithm of the grid-walk parameter, } \\
d L / d \ln (\beta)\end{array}$ & $\frac{d L}{d \ln (\beta)}=b+2 c \ln (\beta)+3 d \ln ^{2}(\beta)=0$ & & $(3.2)$ \\
\hline $\begin{array}{l}\text { Maximum-likelihood estimate of the number } \\
\text { of steps per unit of time, } \beta_{\mathrm{MLE}}\end{array}$ & $\beta_{\mathrm{MLE}}=\exp \left(\frac{-2 c \pm \sqrt{4 c^{2}-12 b d}}{6 d}\right)$ & & (3.3) \\
\hline Corresponding maximum log-likelihood, $L_{\mathrm{MAX}}$ & $L_{\mathrm{MAX}}=a+b \ln \left(\beta_{\mathrm{MLE}}\right)+c \ln ^{2}\left(\beta_{\mathrm{MLE}}\right)+d \ln ^{3}\left(\beta_{\mathrm{MLE}}\right)$ & & (3.4) \\
\hline $\begin{array}{l}\text { Change in log-likelihood relative to the maxi- } \\
\text { mum log-likelihood, } L-L_{\mathrm{MAX}} \text {, which is } \\
\text { also the implicit equation to calculate the } \\
\text { approximate } 95 \% \text { confidence limits for the } \\
\text { grid-walk parameter for a given sample size }\end{array}$ & $L-L_{\mathrm{MAX}}=b \ln \left(\frac{\beta}{\beta_{\mathrm{MLE}}}\right)+c \ln ^{2}\left(\frac{\beta}{\beta_{\mathrm{MLE}}}\right)+d \ln ^{3}\left(\frac{\beta}{\beta_{\mathrm{MLE}}}\right)$ & $=-1.92$ & $(3.5)$ \\
\hline
\end{tabular}

Notes: Only equations for parameter $\beta$, describing the number of steps an organism moves per unit of time, are presented here. With respect to the mortality probability $\mu$, the grid-walk model is identical to the exponential model and the relevant equations given in Table 2 can be applied. 
TABle 4. Dispersal dates and fates of 19 radio-tagged juvenile Mexican Spotted Owls in Arizona (USA), 1994 and 1995 (modified after Ganey et al. [1998]).

\begin{tabular}{|c|c|c|c|c|c|c|}
\hline \multirow{2}{*}{$\begin{array}{c}\text { Owl } \\
\text { number }\end{array}$} & \multirow{2}{*}{$\begin{array}{c}\text { Dispersal } \\
\text { date }\end{array}$} & \multirow{2}{*}{$\begin{array}{l}\text { Last date } \\
\text { known } \\
\text { dispersing }\end{array}$} & \multirow{2}{*}{$\begin{array}{c}\text { No. of days } \\
\text { dispersing, } \\
t_{i}\end{array}$} & \multirow[b]{2}{*}{ Fate $\dagger$} & \multicolumn{2}{|c|}{ Mortality } \\
\hline & & & & & Low & High \\
\hline 628 & 09 Sep 1994 & 21 Oct 1994 & 42 & $\mathrm{P}$ & D & D \\
\hline 659 & 14 Sep 1994 & 03 Nov 1994 & 50 & $\mathrm{~S}$ & D & D \\
\hline 469 & 14 Sep 1994 & 03 Jan 1995 & 111 & RF & $\mathrm{C}$ & $\mathrm{C}$ \\
\hline 918 & 20 Sep 1994 & 20 Oct 1994 & 30 & $\mathrm{P}$ & $\mathrm{D}$ & $\mathrm{D}$ \\
\hline 508 & 20 Sep 1994 & 10 Nov 1994 & 51 & S & D & D \\
\hline 676 & 25 Sep 1994 & 13 Oct 1994 & 18 & $\mathrm{RF}^{*}$ & $\mathrm{C}$ & D \\
\hline 748 & 26 Sep 1994 & 21 Oct 1994 & 25 & $\mathrm{~S}$ & D & $\mathrm{D}$ \\
\hline 728 & 16 Oct 1994 & 28 Nov 1994 & 43 & $\mathrm{P}$ & D & D \\
\hline 809 & 18 Oct 1994 & 07 Nov 1994 & 20 & $\mathrm{RF}^{*}$ & $\mathrm{C}$ & D \\
\hline 620 & 05 Sep 1995 & 16 Jan 1996 & 133 & $\mathrm{RF}^{*}$ & $\mathrm{C}$ & D \\
\hline 857 & 12 Sep 1995 & 26 Apr 1996 & 227 & SL & $\mathrm{C}$ & $\mathrm{C}$ \\
\hline 640 & 18 Sep 1995 & 02 Oct 1995 & 14 & SL & $\mathrm{C}$ & $\mathrm{C}$ \\
\hline 660 & 20 Sep 1995 & 10 Oct 1995 & 20 & A & A & A \\
\hline 697 & 23 Sep 1995 & 03 Oct 1995 & 10 & SL & $\mathrm{C}$ & $\mathrm{C}$ \\
\hline 838 & 28 Sep 1995 & 05 Feb 1996 & 130 & $\mathrm{P}$ & D & D \\
\hline 678 & 30 Sep 1995 & 20 Nov 1995 & 51 & SL & $\mathrm{C}$ & $\mathrm{C}$ \\
\hline 599 & 06 Oct 1995 & 10 Oct 1995 & 4 & $\mathrm{RF}^{*}$ & $\mathrm{C}$ & D \\
\hline 877 & 06 Oct 1995 & 11 Oct 1995 & 5 & SL & $\mathrm{C}$ & $\mathrm{C}$ \\
\hline 799 & 09 Oct 1995 & 16 Nov 1995 & 38 & $\mathrm{P}$ & D & D \\
\hline
\end{tabular}

$\dagger$ Codes of the fates according to the classification of Ganey et al. (1998): $\mathrm{S}=$ observed death presumably due to starvation, $\mathrm{P}=$ observed death presumably due to predation, $\mathrm{A}=$ successful arrival in a new patch, $\mathrm{SL}=$ radio signal lost, and $\mathrm{RF}=$ radio failure. Radios that were found in burrows, indicating a presumed death of this bird, are marked with an asterisk $\left(\mathrm{RF}^{*}\right)$.

\$Codes for the low- and high-mortality scenario employed in this paper: $\mathrm{D}=$ death, $\mathrm{A}=$ arrived and, $\mathrm{C}=$ censored (fate unknown due to radio loss or failure).

model of this sort. This approach, therefore, allowed us to generalize our conclusions beyond the current data and the current models.

The first equation describes the log-likelihood of the data, given the model and the parameters. From this the (partial-)derivative of the log-likelihood for each parameter is determined (second equation). To find the parameters for which the likelihood of the data is maximized, given the data, these derivates are set to zero and the equation is solved for each parameter (third equation). Filling these optimized parameter values in the general-likelihood equation results in an expression for the maximum log-likelihood (fourth equation). Subtraction of the maximum log-likelihood equation from the general log-likelihood equation results in an expression for the relative change in log-likelihood as a function of the model parameters (fifth equation). By setting the latter equation equal to $-\chi_{0.05[1]}^{2} / 2=-1.92$ (Hilborn and Mangel 1997:164) an implicit equation for the confidence limits of the model parameters is obtained for the current data, or hypothetical larger data sets. For the two simpler models these approximate confidence limits, based on standard likelihood theory, could be checked against exact analytical confidence limits.

\section{The event-based model}

In the event-based model, we looked only at the final fate of each of the 19 birds in the spotted owl data of Ganey et al. (1998) that were observed to initiate dis- persal. We discriminated among three fates: death (Ganey et al. 1998, fates S and P), arrival (Ganey et al. 1998, fate A) and being censored due to radio loss or failure (Ganey et al. 1998, fates RF and SL). For the censored birds the biological fate (death or arrival) is, of course, unknown and these birds, therefore, do not contain biological information in terms of the eventbased model. To the numbers of birds with the two fates, death $(D)$ and arrival $(A)$, a binomial model was fitted. We modeled the probability of arrival with a binomial probability $\rho$ and thus the probability of death as $(1-\rho)$. The specifics of each of the five equations for the event-based model are given Table 1. Exact confidence limits for the binomial parameter $\rho$ can be calculated using the formulas given by Rosner (1995: 177).

\section{The temporally explicit model}

The simplest temporally explicit model of death and arrival is an exponential model with a probability of arriving, $\alpha$, and a probability of dying, $\mu$, both of which are constant over time. (This approach is closely related to the widely used Kaplan-Meier test, but the KaplanMeier test allows for the survival rate to change from one time interval to the next; for details on the KaplanMeier method see Pollock et al. [1989]). Obviously, next to these independent competing risks of death and arrival, the owls in the sample of Ganey et al. (1998) face a third risk, $\gamma(t)$, representing the probability of a bird being censored due to radio loss or failure at mo- 
ment $t$. It can be shown that the maximum-likelihood estimation of $\alpha$ and $\mu$ is independent of the shape of function $\gamma(t)$. There is an intuitive explanation for this independence. In a temporally explicit model each bird contributes to the likelihood of the data, given the model, with two components. The first component accounts for the probability of the specific event (dead, censored, or arrived) that happened at time $t$ and the second component accounts for the probability that none of these three events have happened until time $t$. To get the overall log-likelihood of the data, the natural logarithms of both probabilities per individual are summed over all individuals. This results in a log-likelihood equation that contains no cross-products between the parameters describing the probability of dying, being censored, or arriving, and the values of these parameters under which the likelihood is maximized are thus independent.

The fact that the log-likelihood equation contains the probabilities that nothing has happened until time $t$ implies that all 19 birds in the sample of Ganey et al. (1998) contain useful information for parameterizing the exponential model. For all $N$ birds, including those that were censored, we know that during the time interval $t$ between initiation of dispersal and the moment they were lost due to death, arrival, radio loss, radio failure, or signal loss, the birds did not arrive or die. Next, we have $A$ arrived birds and $D$ dead birds. The specifics of each of the five equations for the temporally explicit model are given Table 2. Exact confidence limits for the parameters $\alpha$ and $\mu$ can be obtained by applying the formulas of Cox and Oakes (1984).

\section{The spatially and temporally explicit model}

We used a random grid-walk dispersal model with patches of suitable habitat as a model that takes both time and space into account. This type of model has been used for many years to study arrival probabilities of animals on a landscape (e.g., Kitching 1971). Ruckelshaus et al. (1997) used a model similar to Kitching's as a test case for the usefulness of spatially explicit dispersal models. In the absence of information on the actual landscape in which the field study took place we used in this comparative study on uncertainty in dispersal models an artificial landscape of $120 \times 120$ grid cells with randomly distributed $4 \times 4$ cell patches of suitable habitat. We examined two levels of suitable habitat, $1 \%$ and $2 \%$. Patches were separated by at least one cell of unsuitable habitat. In a conservation study on the Mexican Spotted Owl, more realistic landscape information would be a necessity, of course.

Organisms were started in a suitable patch and moved randomly over the grid (in eight directions) until they reached another patch of suitable habitat. During this process they had a constant probability of dying, as in the exponential model. As explained above (see Temporally explicit model), the maximum-likelihood estimators of the parameters related to mortality are independent of those related to the competing risk of arrival. Therefore we could use the mortality parameters obtained for the exponential model directly for the random grid-walk model.

A series of 1000000 repetitive simulations of dispersing organisms with the model resulted in a frequency distribution, represented here as vector $\mathbf{n}_{s}$, of the number of organisms that arrived after a given number of steps, $s$. From this distribution a vector representing the fraction of organisms that have initiated dispersal but not yet arrived after a given number of steps, vector $\mathbf{s}_{s}$, was obtained by setting $\mathbf{s}_{0}$ to 1 and sequentially applying $\mathbf{s}_{s}=\mathbf{s}_{s-1}-\mathbf{n}_{s} / 1000000$. Entries in $\mathbf{S}_{s}$ represent the fraction of organisms that is still at risk and are comparable to the quantity $e^{-\alpha t}$ in the exponential model. Finally, the probability of arriving, given the number of steps an organism has moved so far, vector $\mathbf{a}_{s}$, was calculated as $\mathbf{a}_{s}=\ln \left(\mathbf{s}_{s-1} / \mathbf{s}_{s}\right)$. Entries in $\mathbf{a}_{s}$ are comparable with parameter $\alpha$ in the exponential model. But, whereas the probability of arrival in the exponential model was a constant over time, the probability of arrival in the random grid-walk model varies with the number of steps already taken by the animal, due to the specific spatial configuration of the landscape. A description of $\mathbf{s}_{s}$ and $\mathbf{a}_{s}$ would be sufficient to calculate the likelihood of a given data set if we knew the numbers of steps taken by the monitored dispersing organisms to reach patches. The field data, however, are time based and not step based, so we need a parameter, $\beta$, that describes the number of steps an individual moves per unit of time. Moments in time can be converted into numbers of steps with $s=\beta t$.

We are now in the position to calculate the log-likelihood of a given sample with $A$ arrivals out of a total of $N$ tagged individuals. Details of the log-likelihood equation and the other equations for the spatially and temporally explicit model are given in Table 3. Note the resemblance between Eqs. 2.1 and 3.1, keeping in mind that $\mathbf{a}_{\beta t}$ and $\mathbf{s}_{\beta t}$ in Eq. 3.1 represent quantities that are comparable with $\alpha$ and $\mathrm{e}^{-\alpha t}$, respectively, in Eq. 2.1. The term $\mathbf{a}_{\beta t}$ in Eq. 3.1 needs to be multiplied by $\beta$ to convert it from per step to per unit of time. Since $\mathbf{s}_{\beta t}$ is dimensionless, no such conversion is necessary here. Due to the fact that $\mathbf{a}_{s}$ and $\mathbf{s}_{s}$ come from a stochastic model and due to the rounding errors that are introduced by using discrete step values instead of continuous time values, the change in log-likelihood as a function of the number of steps per unit of time showed a slightly noisy pattern, with multiple small local minima around the best estimate of $\beta$. To eliminate this noise, and to proceed from here with analytical techniques, we fitted a third-order polynomial to the loglikelihood profile as a function of $\ln (\beta)$. For the sake of mathematical simplicity, the other four formulas were expressed in terms of this approximation to the log-likelihood profile of parameter $\beta$. 
TABLE 5. Maximum log-likelihoods, best-fitting parameters, and relative 95\% confidence limits of the binomial, exponential, and random grid-walk models.

\begin{tabular}{|c|c|c|c|c|c|c|}
\hline Parameter $\dagger$ & $\begin{array}{l}\text { Mortality } \\
\text { scenario }\end{array}$ & $\begin{array}{l}\text { Maximum } \\
\text { log- } \\
\text { likelihood }\end{array}$ & $\begin{array}{l}\text { Rel. } 95 \% \text { lower } \\
\text { confidence } \\
\text { limit }(\%)\end{array}$ & Best fit & $\begin{array}{l}\text { Rel. } 95 \% \text { upper } \\
\text { confidence } \\
\text { limit }(\%)\end{array}$ & $\begin{array}{l}\text { Calculation } \\
\text { method }\end{array}$ \\
\hline \multicolumn{7}{|c|}{ Success-related parameters } \\
\hline Binomial $\rho$ & low & $-3.139^{1.4}$ & $6.0^{1.5 \mathrm{a}}$ & $0.111^{1.3}$ & $365^{1.5 a}$ & approx. \\
\hline Binomial $\rho$ & low & $-3.139^{1.4}$ & $2.5^{\text {Ros }}$ & $0.111^{1.3}$ & $434^{\text {Ros }}$ & exact \\
\hline Binomial $\rho$ & high & $-3.525^{1.4}$ & $5.9^{1.5 a}$ & $0.0769^{1.3}$ & $386^{1.5 a}$ & approx. \\
\hline Binomial $\rho$ & high & $-3.525^{1.4}$ & $2.5^{\operatorname{Ros}}$ & $0.0769^{1.3}$ & $468^{\text {Ros }}$ & exact \\
\hline \multicolumn{7}{|c|}{ Arrival-related parameters } \\
\hline Exponential $\alpha$ & $\ldots$ & $-7.930^{2.4 \mathrm{~A}}$ & $5.7^{2.5 a}$ & $0.000979^{2.3 a}$ & $440^{2.5 a}$ & approx. \\
\hline Exponential $\alpha$ & $\ldots$ & $-7.930^{2.4 \mathrm{~A}}$ & $2.6^{\operatorname{Cox}}$ & $0.000979^{2.3 a}$ & $369^{\operatorname{Cox}}$ & exact \\
\hline Grid-walk $\beta$ (1\%) & $\ldots$ & $-7.732^{3.4}$ & $10.4^{3.5}$ & $1.317^{3.3}$ & $535^{3.5}$ & approx. \\
\hline Grid-walk $\beta(2 \%)$ & $\cdots$ & $-7.767^{3.4}$ & $11.6^{3.5}$ & $0.671^{3.3}$ & $460^{3.5}$ & approx. \\
\hline \multicolumn{7}{|c|}{ Mortality-related parameters } \\
\hline Exponential $\mu$ & low & $-46.80^{2.4 \mathrm{D}}$ & $45.7^{2.5 b}$ & $0.00783^{2.3 b}$ & $186^{2.5 b}$ & approx. \\
\hline Exponential $\mu$ & low & $-46.80^{2.4 \mathrm{D}}$ & $43.2^{\operatorname{Cox}}$ & $0.00783^{2.3 b}$ & $180^{\operatorname{Cox}}$ & exact \\
\hline Exponential $\mu$ & high & $-65.34^{2.4 \mathrm{D}}$ & $53.5^{2.5 b}$ & $0.0117^{2.3 b}$ & $168^{2.5 b}$ & approx. \\
\hline Exponential $\mu$ & high & $-65.34^{2.4 \mathrm{D}}$ & $51.7^{\mathrm{Cox}}$ & $0.0117^{2.3 b}$ & $164 \mathrm{Cox}$ & exact \\
\hline
\end{tabular}

Notes: The superscripts on the data entries refer to the equations used to derive the quantity (see Tables 1-3 for the equations); Ros $=$ Rosner (1995), Cox $=$ Cox and Oakes (1984). Note that the arrival $(A)$-related and the mortality $(D)$ related parts of Eq. 2.4 are used separately.

$\dagger$ Parameters: $\rho=$ probability of arrival; $\alpha=$ probability of arrival; $\beta=$ parameter related to the number of steps an individual moves per unit of time; $\mu=$ probability of dying.

\section{RESULTS}

To compare our three models, we applied each model to the same data set, following the same scenarios used by Ganey et al. (1998): one low-mortality scenario ( $D$ $=8$ ) that censored all data from the 10 owls with unknown fates and another high-mortality scenario $(D=$

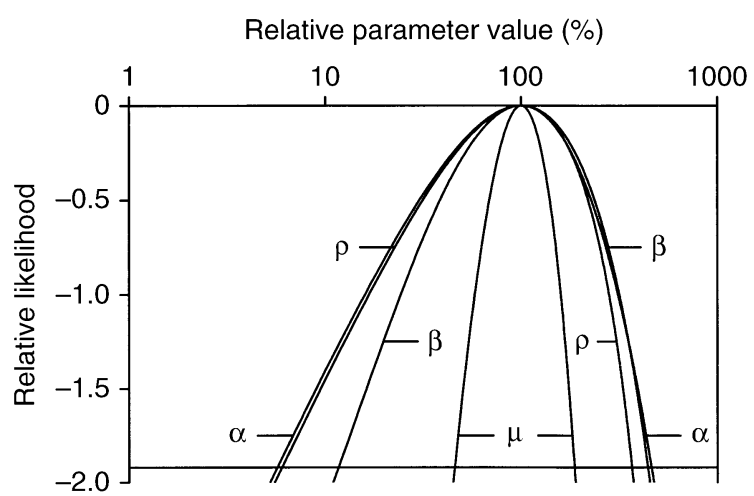

FIG. 1. Log-likelihood profiles for the parameters $\rho$ (probability of arrival) (low mortality) of the binomial; $\alpha$ (probability of arrival) and $\mu$ (probability of dying) (low mortality) of the exponential; and $\beta$ ( $2 \%$ suitable habitat) of the random grid-walk model (Eqs. 1.5, 2.5a/b, 3.5, respectively). Loglikelihoods are scaled by subtracting the maximum log-likelihood from the likelihood for a given parameter value. Parameters are expressed as percentages, relative to the bestfitting parameter $(=100 \%)$. All graphs therefore necessarily have their maximum at the point $(100 \%, 0)$. Approximate confidence limits for the data of Ganey et al. (1998) for the Mexican Spotted Owl were obtained by calculating the values of the parameters for which the log-likelihood was 1.92 worse than the maximum likelihood.
12) that assumed four of the birds with unknown fate to be actually dead, because the radio transmitters were found in burrows (Ganey et al. 1998:213). For details see Table 4.

\section{The event-based model}

An overview of all the results for the relevant parameters for the three models is given in Table 5. To make the confidence limits comparable among models, they were expressed as a percentage of the best fitting parameter and referred to as "relative confidence limits." Obviously, under the low-mortality scenario the best fitting binomial parameter $\rho_{\mathrm{MLE}}$ equals 0.111 and under the high-mortality scenario $\rho_{\mathrm{MLE}}=0.0769$. Under the low-mortality scenario the approximate relative lower and upper $95 \%$ confidence limits are $6.0 \%$ and $365 \%$, respectively (Fig. 1). Relative confidence limits under the high-mortality scenario are very comparable, $5.9 \%$ and $386 \%$, respectively. In Fig. 1 these values correspond to the projections on the $x$-axis of the intersections of the horizontal line at -1.92 and the loglikelihood profile of the event-based model (marked with $\rho$ ). In Table 5 also the exact relative confidence limits that result from Rosner's (1995) formula are given. These are somewhat wider than those derived by the likelihood approximation. Under both methods, and for both mortality scenarios, the relative lower limits, being 20-40 times smaller than the best-fitting values, are wide. Relative upper limits, being 3.7-4.7 times larger than the best-fitting values, are also wide, but much narrower than then the lower limits.

In addition to this analysis we also examined the development of the relative confidence limits for sam- 


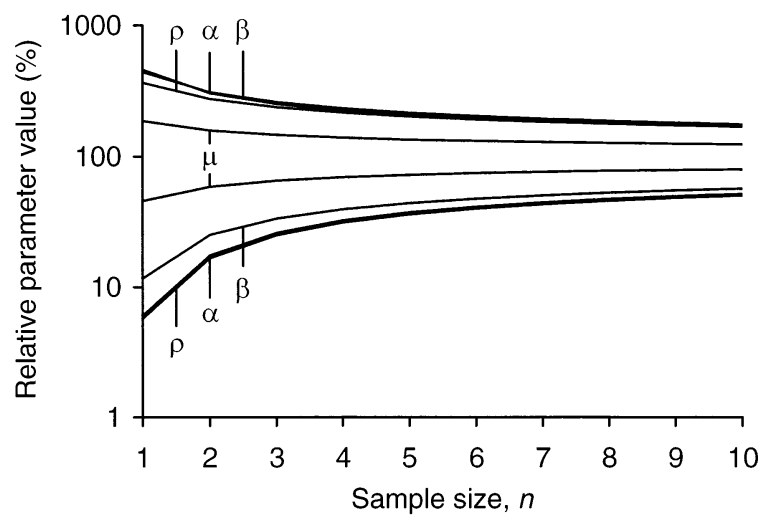

FIG. 2. Relative confidence limits for the parameters $\rho_{\text {MLE }}$ (low mortality) of the binomial model, $\alpha_{\mathrm{MLE}}$ and $\mu_{\mathrm{MLE}}$ (low mortality) of the exponential model, and $\beta_{\mathrm{MLE}}(2 \%$ suitable habitat) of the random grid-walk model as a function of sample size $n$. Parameters are expressed as percentages, relative to the best-fitting parameter $(=100 \%)$. Sample sizes, $n$, are expressed relative to the size of the data set of Ganey et al. (1998) for the Mexican Spotted Owl. The lines are based on approximate confidence limits obtained by calculating the parameter values for which the log-likelihood is $1.92 / n$ worse than the maximum log-likelihood.

ple sizes of up to 10 times the amount of data collected by Ganey et al. (1998). For this purpose we defined a variable $n$ that we used as a multiplier to the numbers of owls that were dead $(D)$, censored $(C)$ or had arrived $(A)$ in the data of Ganey et al. Thus, for the original data $n=1, A=1$, and $D=8$ (low mortality) or 12 (high mortality), while for a sample size of 10 times the amount collected by Ganey et al. $n=10, A=10$, and $D=80$ or 120 .

The results (Fig. 2) show that there is a rapid gain in confidence if a second successful owl $(n=2)$ had been observed: the lower limit increases from $2.5 \%$ to $12.4 \%$ of the best-fitting value, while the upper limit decreases from 4.3 times larger to 3.1 times larger than the best-fitting value. Sample sizes of three $(n=3)$ to four times $(n=4)$ the data of Ganey et al. (1998) result in a further narrowing of the confidence limits, but this process rapidly slows down above these sample sizes and, with respect to confidence limits, there seems little use in sampling more than 60-80 owls, assuming that this will result in 3-4 successful owls.

\section{The temporally explicit model}

Again, we fitted the two parameters, the arrival probability $\alpha$ and the mortality probability $\mu$, of the temporally explicit model to the owl data of Ganey et al. (1998) for the low- and the high-mortality scenarios. In both scenarios the best-fitting value for $\alpha_{\mathrm{MLE}}=$ 0.000979 . The best fitting values for $\mu_{\mathrm{MLE}}=0.00783$ and 0.0117 , respectively, for the low- and high-mortality scenarios. The approximate relative lower and upper $95 \%$ confidence limits of the arrival probability $\alpha_{\text {MLE }}$ are $5.7 \%$ and $364 \%$ of the best-fitting value, re- spectively. Note that these relative confidence limits are close to those that were obtained for the binomial parameter $\rho_{\text {MLE }}$. Exact confidence limits of parameters calculated with the formulas of Cox and Oakes (1984) are somewhat wider (Table 5). The approximate relative $95 \%$ confidence limits of the mortality probability $\mu_{\text {MLE }}$ under the lower mortality scenario are $45.7 \%$ and $186 \%$ and under the high-mortality scenario $53.5 \%$ and $168 \%$. These approximate limits are close to the exact limits (Table 5). For both mortality scenarios the relative confidence around the mortality probability $\mu$ are much narrower than that around the arrival probability $\alpha_{\mathrm{MLE}}$. This makes sense, as the data of Ganey et al. (1998) contain only one observation of a successful arrival but $8-12$ observations of death.

Analysis of the development of the relative confidence limits of the temporally explicit model for larger samples sizes (Fig. 2) shows that for the arrival probability, $\alpha_{\mathrm{MLE}}$, the decrease of the confidence interval closely follows the results obtained for the binomial parameter $\rho_{\text {MLE }}$. Thus, with the exponential model we have to conclude that from the point of view of the confidence limits again there seems little use in sampling more than $60-80$ owls, assuming that about 3-4 owls will be successful. The development of the relative confidence limits around the mortality probability is of less interest because these limits are already in a narrower range.

\section{The spatially and temporally explicit model}

The random grid-walk model resulted in a probability of arriving after a given number of steps, $\mathbf{a}_{s}$, for both the $1 \%$ and the $2 \%$ suitable habitat map (Fig. 3). Using this distribution, we derived for each habitat scenario the likelihood of Ganey et al.'s (1998) data (Fig. 4) with respect to arrival-related parameter $\beta$ of the randomgrid walk model. The fit of the third-order polynomial

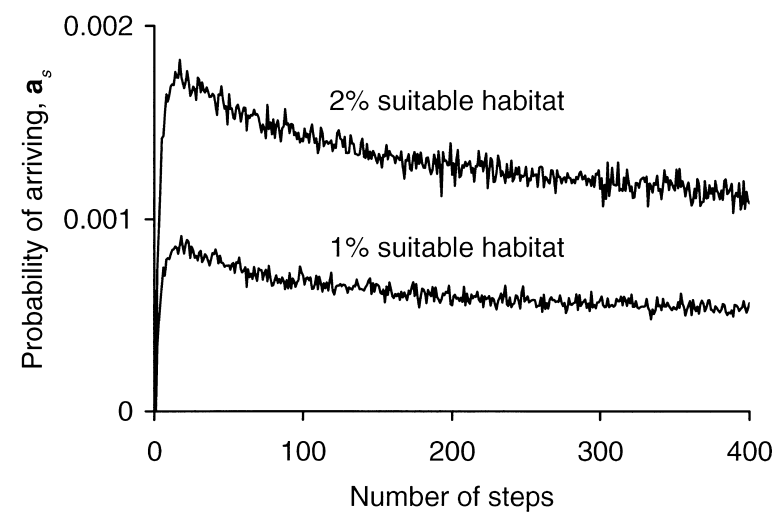

FIG. 3. Probability of arriving after a given number of steps, $\mathbf{a}_{s}$, for the $1 \%$ and $2 \%$ suitable-habitat scenario in the random grid-walk model. This quantity is comparable with parameter $\alpha$ of the exponential model. These probabilities were obtained for each scenario by simulating 1000000 dispersing individuals until they arrived in a patch. 


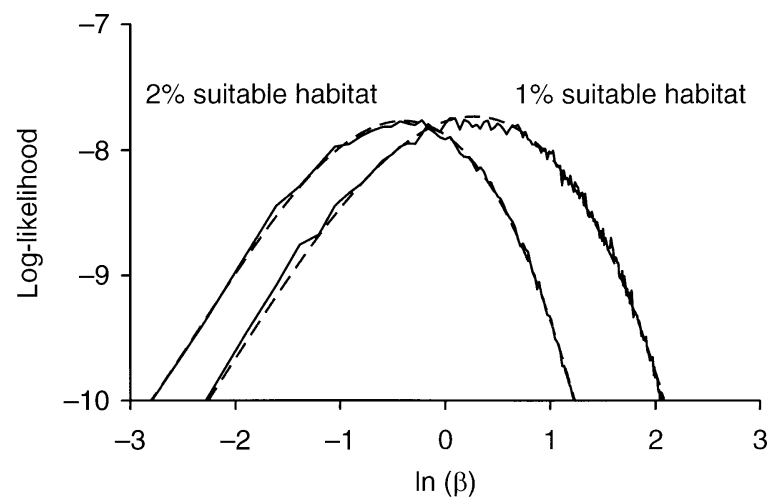

FIG. 4. Log-likelihood profile for the natural logarithm of parameter $\beta$ of the random grid-walk model for the $1 \%$ and $2 \%$ suitable-habitat scenario (solid lines; Eq. 3.1a). Third-order polynomial trend lines that were fitted to the original log-likelihood profile are also shown (dashed lines; Eq. 3.1b).

approximation to this log-likelihood profile was excellent, with $R^{2}=99.5\left(L=-0.078 \ln ^{3}(\beta)-0.487 \ln ^{2}(\beta)+\right.$ $0.286 \ln (\beta)-7.771)$ for the $1 \%$ suitable habitat scenario and $R^{2}=99.8\left(L=-0.112 \ln ^{3}(\beta)-0.790 \ln ^{2}(\beta)\right.$ $-0.577 \ln (\beta)-7.879)$ for the $2 \%$ suitable habitat scenario (Fig. 4). Best-fitting values for the number of steps per unit of time, $\beta_{\mathrm{MLE}}$, derived from the polynomial approximation to the log-likelihood profile were 1.3165 and 0.6708 for the $1 \%$ and $2 \%$ suitable habitat, respectively. As explained earlier, the mortality parameters of the spatially explicit dispersal model could be taken directly from the analysis with the exponential model: $\mu_{\mathrm{MLE}}=0.00783$ (low mortality) and $\mu_{\mathrm{MLE}}=$ 0.0117 (high mortality).

For the $1 \%$ suitable habitat map, the approximate relative $95 \%$ confidence limits of parameter $\beta_{\text {MLE }}$ were $10.4 \%$ and $535 \%$ of the best-fitting values. For the $2 \%$ suitable habitat map these values were $11.6 \%$ and $460 \%$. The relative upper limits were thus of the same order as those that were obtained for the binomial prob- ability $\rho_{\text {MLE }}$ of the event-based model and the constant probability of arrival $\alpha_{\text {MLE }}$ of the temporally explicit model. The lower limits of the spatially and temporally explicit model were narrower than those obtained for the two simpler models, however. The reason for this difference can be found in the shape of the curves describing the probability of arriving in a given step (Fig. 3). For the earliest steps these probabilities are low, thus making small values of $\beta$ unlikely. Thus, the structure of the spatially explicit model has a positive effect on the certainty with which we can estimate its parameters.

The development of the approximate relative confidence limits of $\beta_{\mathrm{MLE}}$ with increasing sample size follows the same decrease as was observed for the binomial probability $\rho_{\text {MLE }}$ of the event-based model and the probability of arrival $\alpha_{\mathrm{MLE}}$ of the temporally explicit model (Fig. 2). For the lower limit, the initial confidence limit at $n=1$ was narrower than those obtained for $\rho_{\mathrm{MLE}}$ and $\alpha_{\mathrm{MLE}}$ and this difference remained intact for larger values of $n$. Also for $\beta_{\mathrm{MLE}}$ we concluded that the level of confidence decreases only slowly for values of $n$ greater than $n=4$ or 5 .

\section{Comparing overall dispersal success}

So far we reported on the best-fitting values and confidence limits of each of the model parameters ( $\rho$, $\alpha, \mu, \beta)$ separately. The aim of all models, however, is to predict dispersal success, and one would therefore like to compare the models with respect to the uncertainty in this ecologically relevant parameter. To distinguish among the models in this comparison we will refer to dispersal success resulting from the binomial, exponential, and random-grid walk model as $\rho_{\mathrm{BIN}}, \rho_{\mathrm{EXP}}$ and $\rho_{\text {GRID }}$, respectively (Table 6 ). Best-fitting values and confidence limits $\rho_{\text {BIN }}$ have already been reported for both mortality scenarios. The best-fitting values of dispersal success for the exponential model necessarily match those of the event-based model since $\rho_{\mathrm{EXP}, \mathrm{MLE}}=$ $\alpha_{\mathrm{MLE}} /\left(\alpha_{\mathrm{MLE}}+\mu_{\mathrm{MLE}}\right)=A /(A+D)=\rho_{\mathrm{BIN}, \mathrm{MLE}}$. A first

TABLE 6. Best-fitting parameters and relative $95 \%$ confidence limits of overall dispersal success as calculated with the binomial, exponential, and random grid-walk models (with $1 \%$ or $2 \%$ suitable habitat).

\begin{tabular}{|c|c|c|c|c|}
\hline Model & Parameter $\dagger$ & $\begin{array}{l}\text { Rel. 95\% lower } \\
\text { confidence } \\
\text { limit }(\%)\end{array}$ & Best fit & $\begin{array}{l}\text { Rel. 95\% upper } \\
\text { confidence } \\
\text { limit }(\%)\end{array}$ \\
\hline \multicolumn{5}{|c|}{ Low-mortality scenario } \\
\hline Binomial & $\rho_{\mathrm{BIN}}$ & 6.0 & 0.111 & 365 \\
\hline Exponential & $\rho_{\mathrm{EXP}}$ & 4.0 & 0.111 & 444 \\
\hline Grid-walk (1\%) & $\rho_{\text {GRID }}$ & 6.7 & 0.100 & 518 \\
\hline Grid-walk (2\%) & $\rho_{\text {GRID }}$ & 5.7 & 0.112 & 420 \\
\hline \multicolumn{5}{|c|}{ High-mortality scenario } \\
\hline Binomial & $\rho_{\text {BIN }}$ & 5.9 & 0.0769 & 386 \\
\hline Exponential & $\rho_{\mathrm{EXP}}$ & 4.1 & 0.0769 & 477 \\
\hline Grid-walk (1\%) & $\rho_{\text {GRID }}$ & 6.1 & 0.0716 & 525 \\
\hline Grid-walk (2\%) & $\rho_{\mathrm{GRID}}$ & 5.0 & 0.0785 & 448 \\
\hline
\end{tabular}

$\dagger$ Dispersal success for each of the models is referred to as $\rho$ with the appropriate subscript. 
approximation to the upper confidence limits of $\rho_{\text {EXP,MLE }}$ was obtained by combining the upper limit of the arrival probability with the lower limit of the mortality probability, $\rho_{\text {EXP,upp }}=\alpha_{\text {upp }} /\left(\alpha_{\text {upp }}+\mu_{\text {low }}\right)$, and vice versa for the lower limit, $\rho_{\text {EXP,low }}=\alpha_{\text {low }} /\left(\alpha_{\text {low }}+\mu_{\text {upp }}\right)$. The relative $\log$-likelihood (Eq. 2.5a/b) associated with these values of $\rho_{\text {EXP,upp }}$ and $\rho_{\text {EXP,low }}$, however, would of course be $2 \times-1.92=-3.84$. This violates the principle that the drop in likelihood due to two parameters should not exceed $-\chi_{0.05[2]}^{2} / 2=-3.00$ (Hilborn and Mangel 1997:173). The intuitive argument here is that it is unlikely that both parameters are at the edge of their uncertainty range at the same time. We therefore have an extra constraint on the uncertainty in $\rho_{\mathrm{EXP}}$, in addition to the independent (see Methods, above, for the temporally explicit model) single-parameter constraints on the uncertainty in $\alpha$ and $\mu$. We checked for the minimum and maximum value $\rho_{\mathrm{EXP}}$ could take within these constraints (Table 6). This analysis shows that the approximate relative $95 \%$ confidence limits of parameter $\rho_{\text {EXP,MLE }}$ are slightly wider than those of $\rho_{\text {BIN,MLE }}$. Also, for the random grid-walk model we checked for the minimum and maximum value $\rho_{\mathrm{GRID}}$ within these constraints discussed above. Approximate relative upper $95 \%$ confidence limits were again slightly larger than those obtained for the binomial model. Lower $95 \%$ confidence limits were close to or even smaller than those obtained for the binomial model. These results show that there mostly is a slight increase in uncertainty in dispersal success when moving from a one-parameter to a two-parameter model, but that this need not always be the case.

\section{DISCUSSION}

We set out in this study to disentangle the effects of model type on the confidence we should have in dispersal parameters estimated from data on dispersing animals. We did so by fitting three models: an eventbased binomial model, a temporally explicit exponential model, and a spatially and temporally explicit random grid-walk model. To link the spatial dynamics with the temporal dynamics we introduced a parameter $\beta$ representing the number of spatial units an organism moves per unit of time. We used two mortality scenarios (low and high) and, in the case of the spatially explicit model, two landscape descriptions (1\% and $2 \%$ suitable habitat). By using the common statistical approach of maximizing the likelihood of the data given the model (sensu Hilborn and Mangel 1997), we calculated best-fitting values for each of these parameters (Table 5). Using the same approach, we derived approximate confidence limits around these best-fitting values (Table 5). Finally, the likelihood approach allowed for a simple extrapolation of these approximate confidence limits, if larger sample sizes were available (Fig. 2).

As a concrete example, we used the Mexican spotted owl data of Ganey et al. (1998) on 19 radio-tagged dispersing birds. We found a large discrepancy between the Mexican spotted owl parameters and their confidence limits, derived here, and those that were originally derived by Ganey et al. (1998). Ganey et al.'s survival rates were always higher, going up to $55 \%$, as opposed to a highest value of $11 \%$ successful dispersers found in our present study. Confidence limits given by Ganey et al. were always wider than the ones that are given here. These contradictions are only superficial, however. To check this for ourselves, we repeated the Kaplan-Meyer analysis that Ganey et al. performed on their data and were able to reproduce their results. This exercise clarified what the underlying differences are between the two analyses. The survival rates that Ganey et al. reported relate to the survival during the dispersal process, but not to the probability that a disperser successfully makes it to a new patch. In the Kaplan-Meyer survival analysis, this one successful bird was simply treated as a bird that did not die, not as a bird that did arrive. In fact, arrival probability was not dealt with at all in the analysis of Ganey et al. With respect to the uncertainty in survival, the wider limits that resulted from the Kaplan-Meyer analysis can be understood if one realizes that in fact the Kaplan-Meyer procedure is a relatively parameter-rich model, because it estimates for each time interval an independent survival rate. This results in a very flexible model with respect to the pattern of survival, but at the cost of a higher uncertainty in the overall survival. In the analysis presented here we chose to estimate only one constant mortality rate for the whole period of two years. This procedure resulted in a higher accuracy of our estimate of long-term survival at the cost of a lack of information on small-scale temporal variation in survival.

The most striking result of our analysis is the similarity in uncertainty of parameters $\rho_{\mathrm{MLE}}$ (the maximumlikelihood estimate of the binomial-model probability of success), $\alpha_{\text {MLE }}$ (the exponential-model probability of arrival), and $\beta_{\text {MLE }}$ (the number of steps per unit of time in the random grid-walk model) (Fig. 1). This similarity remains if larger sample sizes than those collected by Ganey et al. (1998) had been available (Fig. 2). If we take a close look, it is actually the most complex model that has the narrowest confidence interval around its parameters (Fig. 1). We believe that both phenomena have a general meaning, beyond the specific data and the specific series of models that are used in our analysis. To understand the similarity in uncertainty it is important to realize that the three models are in fact a series of nested models. The simplest of the three, the binomial model is essentially equal to the exponential model but describes the dispersal process in a more lumped fashion with $\rho_{\mathrm{MLE}}=\alpha_{\mathrm{MLE}} /\left(\alpha_{\mathrm{MLE}}+\mu_{\mathrm{MLE}}\right)$. The exponential model is, in turn, a specific case of the more complex random grid-walk model. To mimic the exponential model with the random grid-walk model we would have to perform a random grid-walk on a 
landscape with no patches and an equal probability of arrival $\alpha$ per cell. So, in the transition from the binomial to the exponential model we introduce the concept of mortality and arrival being two separate processes underlying successful dispersal. In the transition from the exponential to the random grid-walk model we introduce the concept of the landscape being patchy. Both concepts are essential to our thinking of the actual system under study and they can be seen as qualitative sources of knowledge that were brought into the model, next to the actual dispersal data.

The effect of introducing the concept of patchiness was that fast arrivals were unlikely (Fig. 3). This in turn led to narrower relative lower confidence limits for the random grid-walk model, as opposed to the other two models. Very low values of parameter $\beta_{\mathrm{MLE}}$, representing the speed at which an organism moves through the landscape, are unlikely because such slow organisms would get stuck between patches and never arrive, whereas the data show that some do arrive. This makes perfect sense. Thus, the qualitative knowledge of the system, in this case patchiness, which could be accommodated by the more complex model, had a positive effect on the confidence interval for the critical parameter, in this case $\beta$.

This improvement is also reflected in the somewhat higher maximum likelihood for the arrival-related parameter of the random-grid walk model, when compared to that component of the exponential model (Table 5). From an information-theoretic point of view (Akaike 1992), the random-walk model should thus be preferred over the exponential model, given the fact that the number of parameters that was estimated on the basis of the field data was equal. But the difference is only small and, given the simplicity of implementing and analyzing the exponential model, we suggest using both models concurrently. The performance of the binomial model cannot be compared with that of the other two models by means of an information criterion since it employs the data in a different way: the binomial model only uses the number of arrived and dead birds, whereas the exponential and the random grid-walk model both also take into account the time at which these events took place.

When looking at the overall dispersal success, as predicted by the three models (Table 6), we saw that there was some increase in uncertainty when we moved from the single-parameter binomial model to the twoparameter exponential model. For the lower confidence limits of the two-parameter grid-walk model, however, this increase in uncertainty was compensated by the better fit of the grid-walk model to the data. This resulted in lower confidence limits for overall dispersal success resulting from the two-parameter grid-walk model that were only slightly worse ( $2 \%$ suitable habitat) or even better ( $1 \%$ suitable habitat) than those of the single-parameter binomial model. Compared with the two-parameter exponential model the more com- plex two-parameter random grid-walk model did better for all lower limits and for the upper limits of the $2 \%$ suitable-habitat scenario.

We think that there is an important general lesson to be learned here. The more basic biological detail we introduce into the model a priori (thus not based on the data that are going to be used in the process of parameter estimation), the likelier the data will be, given the model, and thus the narrower the confidence intervals will be around the model parameters, as long as the number of parameters that is estimated by maximizing the likelihood of the data, given the model, does not increase (or increases only slightly) with model complexity. The fact that the number of calibrated parameters does not necessarily increase with model complexity seems counterintuitive. Mostly people would associate complex models with models that have many free parameters. If this were necessarily true, the amount of available data would indeed set a limit to model complexity, because it is a basic statistical rule that the number of estimated parameters should be (far) less than the number of data points. However, if certain concepts can be brought in a priori, (such as levels of patchiness of a landscape or body-size relationships in the biology of an organism), the number of free parameters in these more complex models that are to be estimated from the actual data by means of statistical techniques can still be limited to a few rate or scaling parameters. The random grid-walk model exemplifies this. For this relatively complex model only two rate parameters were estimated from the field data, one related to exploration of the habitat and one related to mortality.

The strategy proposed here of structuring the model on the basis of the available basic biological information on the organisms and the landscape in which these organisms live, and using the available field data only to estimate a few rate or scale parameters, has another main advantage, beyond being a way to efficiently extract information from these potentially scarce data. This strategy also reduces the potential for error propagation to occur. That errors in the underlying parameters are not magnified by spatially explicit models has already been shown (Mooij and DeAngelis 1999). But even without such a magnification, error propagation could still be a serious problem. In the ideal situation one would have perfect knowledge of every component of the system-in which case a model composed of such components would necessarily produce accurate predictions. However, unlike some physical systems for which this is possible, this is an unreachable goal in ecology. In ecological models we have reasonable to good knowledge for some components but for others we have poor to very poor knowledge. The errors in each of these components may propagate in a model that is built of such components and the predictions made with such a model would therefore necessarily become unreliable. 
In the case of Ganey et al.'s (1998) owl data we had no knowledge of the actual landscape these owls lived in, except that it was patchy (of course we could have contacted Ganey et al. to obtain such data, but producing a realistic owl model is not the purpose of this paper). Based on this limited knowledge we created two patchy-landscape maps, one with $1 \%$ and one with $2 \%$ suitable habitat. Now assume that the $1 \%$ map comes closer to the real owl landscape than the $2 \%$ map. In that case, the estimate of $\beta_{\mathrm{MLE}}=1.317$ that was obtained for the $1 \%$ map comes closer to the real value than the $\beta_{\mathrm{MLE}}=0.671$ that was obtained for the $2 \%$ map. In a study that aims at estimating a specific process rate, in this case $\beta_{\mathrm{MLE}}$, a good description of the landscape is essential to get an unbiased estimate of $\beta_{\text {MLE}}$. But in a study that aims to predict the overall number of successful dispersing owls, the bias in one component would do less harm, as long as it is counteracted by a bias in another component. The combination of $2 \%$ suitable habitat with $\beta_{\mathrm{MLE}}=1.317$ or $1 \%$ suitable habitat with $\beta_{\mathrm{MLE}}=0.671$ both result in the same average dispersal success (Table 6). The same reasoning would apply if, in addition to the different landscape maps, we had checked for different movement strategies (Turchin 1998), in addition to the random-grid walk. This process of counterbalancing the inevitable biases in some components (e.g., landscape maps, movement rules) with deliberate biases in other components is guaranteed by directly estimating a few rate or scale parameters from data at the level of interest, in this case the number of successful dispersers. The need for counterbalancing biases by means of estimating some rate or scale parameters from data at the level of interest will hold for both the simpler models, which are necessarily biased because they average out detail in landscape structure or movement patterns, and for the more detailed models, which could be biased due to a wrong classification of the landscape elements in the model or to wrong movement rules.

Our results indicate that spatially explicit population models that include relevant landscape details may, in fact, be preferable over simpler models that ignore basic information on the system at hand, even in cases where specific field data are scarce, as in the case of the Mexican Spotted Owl. Judicious employment of landscape details, movement strategies, and the available field data on dispersal enables spatially explicit population models to serve as important and reliable tools in conservation studies.

\section{ACKNOWLEDGMENTS}

We thank Margaret Hurley and Henriet Nienhuis for statistical advice and Michael McCarthy, Bart Nolet, Peter Schippers, Matthijs Vos and an anonymous reviewer for valuable comments on earlier versions of this manuscript. This study was supported by the co-operative effort of the UnitedStates Geological Survey, Biological Resources Division and the Netherlands Institute of Ecology, Centre for Limnology. DLD was supported in significant part by funding from the Critical Ecosystems Studies Initiative of the U.S. Department of Interior and in part by the USGS's Florida Caribbean Science Center. This is publication 3071 of the NIOO-KNAW Netherlands Institute of Ecology, Centre for Limnology.

\section{Literature Cited}

Akaike, H. 1992. Information theory and an extension of the maximum likelihood principle. Pages $610-624$ in S. Kotz and N. Johnson, editors. Breakthroughs in statistics 1 . Springer Verlag, New York, New York, USA.

Bart, J. 1995. Acceptance criteria for using individual-based models to make management decisions. Ecological Applications 5:411-420.

Cox, D. R., and D. Oakes. 1984. Analysis of survival data. Chapman and Hall, New York, New York, USA.

Doak, D. F., and L. S. Mills. 1994. A useful role for theory in conservation. Ecology 75:615-626.

Franklin, A. B., D. R. Anderson, R. J. Gutiérrez, and K. P. Burnham. 2000. Climate, habitat quality, and fitness in northern spotted owl populations in northwestern California. Ecological Monographs 70:539-590.

Ganey, J. L., W. M. Block, J. K. Dwyer, B. E. Strohmeyer, and J. S. Jenness. 1998. Dispersal movements and survival rates of juvenile Mexican spotted owls in Northern Arizona. Wilson Bulletin 110:206-217.

Grimm, V., K. Frank, F. Jeltsch, R. Brandl, J. Uchmanski, and C. Wissel. 1996. Pattern-oriented modelling in population ecology. Science of the Total Environment 183: 151-166.

Hanksi, I. 1999. Metapopulation ecology. Oxford University Press, Oxford, UK.

Hanski, I., J. Alho, and A. Moilanen. 2000. Estimating the parameters of survival and migration of individuals in metapopulations. Ecology 81:239-251.

Hilborn, R., and M. Mangel. 1997. The ecological detective: confronting models with data. Princeton University Press, Princeton, New Jersey, USA.

Kitching, R. L. 1971. A simple simulation model of dispersal of animals among units of habitats. Oecologia 7:95-116.

Lindenmayer, D. B., M. A. McCarthy, and M. L. Pope. 1999. Arboreal marsupial incidence in eucalypt patches in southeastern Australia: a test of Hanski's incidence function metapopulation model for patch occupancy. Oikos 84:99109.

McCarthy, M. A., D. B. Lindenmayer, and H. P. Possingham. 2000. Testing spatial PVA models of Australian treecreepers (Aves: Climacteridae) in fragmented forest. Ecological Applications 10:1722-1731.

McKelvey, K., B. R. Noon, and R. H. Lamberson. 1993. Conservation planning for species occupying fragemented landscapes. The case of the Northern Spotted Owl. Pages 424-450 in P. M. Kareiva, J. G. Kingsolver, and R. B. Huey, editors. Biotic interactions and global change. Sinauer Associates, Sunderland, Massachusetts, USA.

Mooij, W. M., and D. L. DeAngelis. 1999. Error propagation in spattially explicit population models: a reassessment. Conservation Biology 13:903-933.

Pollock, K. H., S. R. Winterstein, C. M. Bunck, and P. D. Curtis. 1989. Survival analysis in telemetry studies: the staggered entry design. Journal of Wildlife Management 53:7-15.

Pulliam, H. R., J. Dunning, and J. Liu. 1992. Population dynamics in complex landscapes: a case study. Ecological Applications 2:165-177.

Rosner, B. 1995. Fundamentals of biostatistics. Fourth edition. Daxbury Press, Belmont, Massachusetts, USA.

Ruckelshaus, M., C. Hartway, and P. Kareiva. 1997. Assessing the data requirements of spattially explicit dispersal models. Conservation Biology 11:1298-1306.

Ruckelshaus, M., C. Hartway, and P. Kareiva. 1999. Dispersal and landscape errors in spattially explicit population models: a reply. Conservation Biology 13:1223-1224. 
South, A. 1999. Dispersal in spatially explicit models. Conservation Biology 13:1039-1046.

Thulke, H.-H., V. Grimm, M. S. Müller, C. Staubach, L. Tischendorf, C. Wissel, and F. Jeltsch. 1999. From pattern to practice: a scaling-down strategy for spatially explicit modelling illustrated by the spread and control of rabies. Ecological Modelling 117:179-202.

Turchin, P. 1998. Quantitative analysis of movement. Measuring and modeling population redistribution in animals and plants. Sinauer Associates, Sunderland, Massachusetts, USA.
Turner, M. G., G. J. Arthaud, R. T. Engstrom, S. J. Hejl, J. Liu, S. Loeb, and K. McKelvey. 1995. Usefulness of spatially explicit population models in land management. Ecological Applications 5:12-16.

Turner, M. G., Y. G. Wu, W. H. Romme, and L. L. Wallace. 1993. A landscape simulation model of winter foraging by large ungulates. Ecological Modelling 69:163-184.

Vos, C. C., J. Verboom, P. F. M. Opdam, and C. J. F. Ter Braak. 2001. Toward ecologically scaled landscape indices. American Naturalist 183:24-41. 